\title{
List of Tables
}

Table 1.1 Roughness parameters of the characterized surfaces.

Table 1.2 Weibull Modulus $m$ (shape parameter) and the scale parameter to for each gecko and set, on virgin PMMA ( $m_{\text {PMMA }}$ and $\left.t_{\text {opMMA }}\right)$ and on glass $\left(m_{\text {class }}\right.$ and $\left.t_{\text {oGlass }}\right)$.

Table 1.3 Adhesion times on virgin PMMA (Test 1) during the moult, (Test 2) on glass surface, and (Test 3 ) on virgin PMMA not during the moult.

Table 1.4 Weibull Modulus $m$ (shape parameter) and the scale parameter $t_{0}$ for each gecko and set on PMMA2400 ( $m_{\text {PMMA2400 }}$ and $\left.t_{\text {0-PMMA2 } 400}\right)$ and PMMA800 ( $m_{\text {PMMA800 }}$ and $\left.t_{\text {O-PMMA800 }}\right)$.

Table 2.1 Roughness parameters for the three different PMMA $(1,2,3)$ surfaces.

Table 2.2 The gecko's adhesion times on PMMA 1, 2, 3 surfaces. Note that, as an index of the gecko's adhesive ability, we use the Weibull scale parameter $t_{0}$ (in seconds) for the distribution of the detachment/failure $F$ (closely related to its mean value).

Table 3.1 Roughness parameters of the studied PMMA and glass surfaces.

Table 4.1 Experimental values (and the number $N$ of measurements) for the adhesion angles $\alpha_{\mathrm{F}}(\mathrm{A})$ and $\alpha_{\mathrm{T}}(\mathrm{B})$ on different surfaces.

Table 5.1 The roughness parameters, sSF and $F_{\text {radial }}$ of the characterized insect/ surface systems. The values ${ }^{(*)}$ are computed by multiplying the parameter $S q$ by the value 3.6, which was previously calculated as Sq/Ad for sandpapers (Sp) with known Ad on which the roughness parameter $S q$ has been observed. 
Table 6.1 Measured roughness parameters of PS surfaces. Note that samples $7 A_{p}$ and $7 B_{p}$ are used only to evaluate the effects of plasma treatment, whereas $7 A_{t}$ and $14 A_{t}$ are new starting samples for the determination of the effects of thermoforming treatment.

Table 6.2 CA measurements of the tested PS surfaces.

Table 6.3 Wenzel roughness parameters $r$ of PS surfaces.

Table 6.4 Contact angle, sliding angle, sliding volume, and sliding speed of a natural 6-month dried adaxial leaf lotus surface.

Table 7.1 Contact angle (CA), tilting angle (TA), sliding volume (SV), and sliding speed (SS) of lotus leaf (LL), copied lotus leaf (CLL), first silicone copy (C1), flat silicone control surface ( 1 1_control), second PS copy (C2), and flat PS control surface (C2_control).

Table 8.1 List of the caves visited for the collection of the samples with collection date and number of samples.

Table 8.2 The measured stress of each stalk in ascending order.

Table 8.3 The main parameters which may influence tensile testing results: systematics, function, silk-producing glands, temperature and humidity, initial length $\left(l_{0}\right)$ of samples, number of tested threads, selected strain rate, and number of tested samples. Spider nomenclature according to (Platnick, 2011). 